\title{
Living and Dying with Alzheimer's Disease: A Case Presentation
}

\section{Lorena M. De La Rosa}

Marybelle and S. Paul Musco School of Nursing and Health Professions, Brandman University, Irvine, California

Email address:

ldelaros@mail.brandman.edu

To cite this article:

Lorena M. De La Rosa. Living and Dying with Alzheimer's Disease: A Case Presentation. American Journal of Nursing Science. Vol. 4, No. 4, 2015, pp. 212-217. doi: 10.11648/j.ajns.20150404.20

\begin{abstract}
The following is a case presentation on the life of Leslie Bernard Wynne, an individual crippled by Alzheimer's Disease (AD) during the last five years of his life. The case study briefly describes the disease process, discusses the genetic component of late-onset $\mathrm{AD}$ in regards to the apolipoprotein $\mathrm{E}$ (APOE) gene, provides a suggested care plan which spans the course of illness from diagnosis until death, outlines treatment options, highlights common comorbidities, and considers the availability of genetic testing. Recommendations based on best practices and research available are provided throughout and have been integrated into the suggested plan of care. The case study also includes recollections of the family of Mr. Wynne during his illness and exposes the great emotional and physical toll that Alzheimer's takes beyond the diagnosed individual.
\end{abstract}

Keywords: Alzheimer's Disease, APOE Gene, Case Study, Plan of Care, Genetic Counseling

\section{Introduction}

Millions of lives are affected by a new or continuing diagnosis of Alzheimer's Disease (AD) every day in the United States [1]. The toll that this disease is poised to take upon the American landscape has devastating potential: medically, financially and emotionally. It is the personal loss, however - the decline of functional status, memory recall, and ability to recognize loved ones - that is so feared. The care of the individual diagnosed with $\mathrm{AD}$ and their family is a challenging task for medical professionals. Caregivers, nurses, counselors, and physicians work to advise, guide, and comfort those who are faced with a tremendous loss on the horizon the loss of the mind. With no available treatments for this harrowing illness, many are left feeling helpless as they watch their loved ones slip away. However, as advancements in research continue, hope remains for preventative therapies, and healthcare providers become better informed in the care and management of this debilitating disease.

The following case study recounts the life of Leslie Bernard Wynne, the author's grandfather. His battle with Alzheimer's lasted five years before the disease took his life in 2009. Information regarding the typical course of lateonset Alzheimer's Disease, including diagnostic techniques and treatment options, will be utilized as a point of reference for the reader. Considerations in formulating a plan of care and ongoing risks faced by the individual and their family are included. Additionally, a discussion about the genetic link to Alzheimer's and available counseling for the patient and family will be included in this case study. Perhaps most poignantly, the retelling of his illness and decline in condition can be visualized through highlights of his artistic body of work produced throughout his life until the days before his death. Full permission to discuss this case was granted by surviving family.

\subsection{Brief Overview of Alzheimer's Disease}

The pathology of $\mathrm{AD}$ was first recognized by Bavarian psychiatrist and neuropathologist Dr. Aloysius Alzheimer in 1906 [2]. After studying the brain of a deceased woman known to have had unusual mental and behavioral symptoms, Dr. Alzheimer recognized the presence of abnormalities within her brain. The findings led to the determination that physical irregularities co-existed with a brain disorder that featured memory loss and decline in everyday functioning, which was later named Alzheimer's Disease. These abnormalities manifest as an overabundance of proteins that accumulate in and around the neurons of the brain and are the pathological hallmark of AD. The proteins, known as betaamyloid plaques and tau tangles, interfere with the normal transmission of information between neurons and contribute to cell death [3]. 
Dementia, which is the loss of cognitive functioning that affects both mental and behavioral tasks, has a range of causes and diagnoses, with $\mathrm{AD}$ as the leading cause of dementia in older adults [4]. Early symptoms may present as apathy, depression, and trouble recalling names, events, or recent conversations; later symptoms include difficulty communicating, confusion, poor judgement, uncharacteristic behavioral changes, and inability to perform normal activities of daily living [3]. It is believed that multiple risk factors contribute to the development of Alzheimer's, including age, family history, cardiovascular health, education, history of traumatic brain injury, and genetic influences [3]. The age of the individual at the time of diagnosis determines whether the diagnosis is early-onset or late-onset Alzheimer's Disease, with different genes attributable to each type. In late-onset $\mathrm{AD}$, the patient is noted to be symptomatic after the age of 60 [5].

The apolipoprotein E (APOE) gene, found on chromosome 19 , has been noted to play a role in one's risk for developing the disease. There are three alleles of the APOE gene identified as e2, e3, and e4. APOE e 2 is the most rare of the alleles and is known to provide some protection against the disease; APOE e3 is the most common of the alleles and neither increases nor decreases one's risk of inheriting the disease; the APOE e4 allele is found in around $10-15 \%$ of the U.S. population and in $40 \%$ of all people diagnosed with late-onset Alzheimer's Disease [5]. The risk for AD is three-fold for those who've inherited one APOE e4 allele, and approximately twelve-fold for those with two e4 alleles [5]. The APOE protein is expressed in high amounts in the central nervous system (CNS) and is thought to play a role in the function and maintenance of synaptic junctions - that is, in the way that neurons communicate with each other. Pathological research has found a positive correlation between beta-amyloid plaque density and the APOE e4 allele in the autopsies of AD patients [6]. These findings, backed by imaging and CNS studies, indicate that those individuals with the e4 allele have an increased development of beta-amyloid plaque present in the brain during their lives, regardless of an AD diagnosis [6].

It has only been recently that the means by which the APOE gene affects beta-amyloid plaques has been suggested. Some studies have shown APOE's influence on the fibrillogenesis of neurological plaques, as well as both the transport and removal of beta-amyloid plaque in the brain [6]. There is some indication that APOE e4 receptors may also have an effect on the development of AD. Cholesterol metabolism, the growth of nervous system tissue, and the formation of beta-amyloid plaques are noted mechanisms by which receptors could influence the course of Alzheimer's [6].

While a genetic component does exist in late-onset $\mathrm{AD}$, genetic testing is not routinely advised. Testing is typically advised only for those at risk for autosomal dominant Alzheimer's Disease, which is associated with early-onset [7]. When managing a diagnosis of AD in which the APOE gene is suspected, the greatest concern lies with the absence or presence of the e4 allele. However, since the allele is neither necessary nor exclusive to causing $\mathrm{AD}$, the consensus of the medical community holds that genetic testing is not recommended [8].

\subsection{Diagnosis}

Screening for cognitive changes typically begins in the office of one's primary care physician. Patients should be screened for cognitive impairment if an individual, or their family members, express concern about changes in memory or thought processes, if the physician notes marked changes in the patient's cognition, or if the individual is age 80 or older [9]. Currently, there are multiple approaches to the screening process, with no single test to definitively make a diagnosis. Taking a thorough family history and discussing the presence of cognitive or behavioral changes with the individual (or their close family) are important components. Using tools such as the Dementia Screening Indicator and the Mini-Cog neurological exam can assist in the identification of symptoms indicative of dementia. A referral to a specialist, such as a neurologist or gerontologist, may be suggested for some patients who exhibit positive testing results.

Although there is no curative treatment for $\mathrm{AD}$, there are advantages to participating in cognitive screening. The ability to update advanced directives, assisting family with obtaining information and referrals, and the possibility of participation in clinical research studies are some of the benefits [9]. Development of a comprehensive plan of care for a newly diagnosed Alzheimer's patient is potentially the most important benefit of performing the neurological screening. As a patient progresses through the disease course of Alzheimer's, there are changes that can be expected, allowing family and caregivers to prepare before becoming overwhelmed. Including multiple disciplines in the patient's care plan will ensure that most concerns regarding AD will be met during the course of the illness.

In 2011, the National Institute on Aging and the Alzheimer's Association created revised guidelines for use during the diagnosis of AD [3]. The effort yielded the identification of three stages of Alzheimer's Disease: preclinical $\mathrm{AD}$, mild cognitive impairment $(\mathrm{MCI})$ due to $\mathrm{AD}$, and dementia due to $\mathrm{AD}$. In preclinical $\mathrm{AD}$, an individual would have no outward symptoms of Alzheimer's but brain changes specific to the disease would be present. Diagnostic criteria has not been identified for this phase, however, due to the need for additional research [3]. With MCI, the clinical presentation resembles mild alterations in memory and thinking without affecting normal daily activities. In the third phase, dementia due to $\mathrm{AD}$, cognitive impairments are markedly worsening and present an impairment in performing normal daily activities. In the example of the case presentation to follow, the first indication of illness presented itself during the second phase, MCI due to AD. The family of Leslie Wynne did not suspect any issue until symptoms began showing themselves and, even then, they were not typical or the most commonly known variety.

\section{Case Presentation}

In the summer of 2004, Leonard Wynne moved back home. 
His mother needed help with a bizarre new behavior that had just begun to surface with his father, Leslie Wynne. "He started drinking a lot," recounts Leonard (personal communication, May 21, 2015). Wynne was always a man who enjoyed an evening nightcap, but when he began rummaging through the liquor cabinet at all hours and having drinks he never favored in the past, the family became alarmed at this unusual behavior. Wynne would also reminisce about things that happened very early in his life that no one had ever heard him talk about. "I kept hearing these long forgotten stories, probably long buried stories, he had never mentioned before. And he would get very sentimental about it," Leonard states (personal communication, May 21, 2015). A change was manifesting itself in all aspects of Wynne's life, but perhaps most poignantly in his professional work. Born in 1920, Leslie Wynne came to live in California with his family from Indiana. At a young age, he was enthralled with painting and creativity, and began studying art under such talents as Alfred Dewey and Norman Rockwell. At fourteen, Wynne won local recognition for his ability. He continued his artwork through high school, college, and to the South Pacific islands, where he served in World War II. After marrying and starting a family, Wynne worked at an architectural firm by day, and painted landscapes at home in his free time. Soon his private work became prominent, and before long he was able to paint professionally at home, exhibiting his paintings in galleries all across the southwest United States. By the age of 30, he was listed among the Who's Who of American Art. He was famous for his California desert scenes and depictions of Yosemite, rich in detail and striking contrast.

His ability blossomed over the years, and his paintings became more beautiful with his age - until the months leading up to that summer. Slowly, his work began to change. Detail was lost, shadows became indistinguishable, and color began to fade. It was about this time that Wynne was diagnosed with depression. "He went through his blue period, his green period," says Leonard (personal communication, May 21, 2015). Before, rocks were steely, sands bisque, and wildflowers popped in purple, yellow, and orange. Then, as Wynne's behavior began to change, his landscapes became cast in blue, once white clouds turned azure, with roads and rolling hills underneath just a lonesome, beryl sight. His daughter, Leontine, recalled the change stoically. "I remember he would ask me every time I would visit, 'What's wrong with this painting? Does this look right to you?' I would always say 'Yes, Dad, it looks beautiful!'” But, there was no mistaking it: his paintings were not the same. It was heartbreaking for the family to see their patriarch struggling with his art, doubting his ability, and feeling frustrated with each painting he completed. "It was like he couldn't figure out how to make it the color he wanted," Leontine added (personal communication, May 21, 2015).

Some time after the changes with Leslie Wynne began, the family was faced with new, disturbing behaviors. Wynne began to take walks around the neighborhood, unannounced. Several times, when family could not find him, he was brought home by police and Good Samaritans. "He said he was trying to get home. 'I want to go home!' he would always tell us," Leontine recalls (personal communication, May 21, 2015). "He used to try to go out and buy alcohol, too, and he didn't have any money." He was prone to agitation and restlessness throughout the day at this time, as well. The family decided to bring him to his primary physician, who suggested a prescription to help slow the symptoms of his dementia. Unfortunately, Wynne suffered from severe aggression as a side effect, and the family stopped the medication before the first bottle had even been finished 1 . "He was very violent," Leonard says (personal communication, May 21, 2015). "It was dangerous. One time he picked up a lamp and threw it across the room." This behavior ceased after the medication was stopped. No further prescriptions were attempted, by the family's choice.

Years went by and Wynne's paintings became less and less detailed. They came to resemble abstract art more than the classic landscapes he became so noted for. He was able to do fewer things for himself, too, which became a strain on his wife and son, who were the primary caregivers. "There was about a two or three year period before he died that it was really difficult to take care of him," Leontine says (personal communication, May 21, 2015). Wynne's children would come during the week to help when they could, and a part-time caregiver was enlisted to assist in daily hygiene. He would often fixate on names or phrases overheard and repeatedly say them aloud. "Mom always had the news on in the background. One day, there was this story about a woman who poisoned her family. For the next couple days afterwards, when we would bring in food, he would always ask us, 'Is this food poisoned?! I'm not going to eat this food!"' Leonard chuckles, recalling the time (personal communication, May 21, 2015). When he began ingesting his oil paints, Leonard made the decision to pack up his palette. In its place came a pad of paper and colored pencils. "He would mix in words to what he was doing, like he was picking up snippets of conversations or thoughts and jotting them down," Leontine remembers (personal communication, May 21, 2015). Indeed, upon examining the hundreds of pages of colored pencil work Wynne completed, one can find such phrases as St. Patrick's Day or I love my family or I'm dying peppered within layers of line scribblings that made no image.

Wynne was hospitalized twice during the course of his Alzheimer's. The first time he collapsed and was found to have pneumonia. During the second hospitalization, he was treated for dehydration and then discharged home. From that time forward, however, he became bedridden. Losing the ability to walk, Wynne had to be carried around the house by his son and caretaker whenever necessary. He was no longer able to participate in meaningful conversation or normal activities, and was unable to recognize family members, though they would sometimes get a glimpse of the man they once knew. "He would lay in his bed and it looked like he was painting,"

1 The specific medication could not be recalled, though the now discontinued Cognex was noted to have this side effect. 
says Leonard (personal communication, May 21, 2015). Arm raised, hand gripping a nonexistent paintbrush, Wynne made delicate brush strokes in the air as his head moved back and forth, from invisible reference photo to canvas. If moved to a chair, he would continue to make the gestures of painting in front of him with great care and concentration, creating another stunning landscape that only he could see. The simple task of feeding himself became confused with the motions of painting, mixing his scrambled eggs and raspberry jelly together as if blending for the perfect color to work with.

The drive to paint remained with Wynne up until his final hours of life. As hospice was called in, just days before his death, his feeble hand continued to dance across the wall near his bed, trying to complete just one last piece. The once robust, kind-hearted patriarch was but a shadow of his former self in the twilight, his mind and body ravaged by untreatable illness. No longer able to eat or drink, comfort measures were started and a bedside vigil of family members began. Quietly, in the early morning hours of November 15, 2009, Wynne passed away in his home surrounded by those that loved him most.

\section{Discussion}

Continuing awareness about $\mathrm{AD}$ in the media and throughout one's medical care received as an aging individual can assist those that may be at risk for developing Alzheimer's to receive the care and counseling they require. A great benefit to an early diagnosis is the ability to implement a comprehensive care plan that can help the patient, family, and caregivers to best manage the progression of disease. This care plan, which should include a variety of healthcare disciplines, can include pharmacological treatments, provide thorough education and teaching about $\mathrm{AD}$, and assist with obtaining help and resources while managing the illness. During the early stages of Alzheimer's, the patient may still be relatively independent and not require intensive caregiving needs. It is necessary for the care plan to reinforce the importance of presence and companionship of family and loved ones during this early time in the diagnosis. Supportive needs may include assistance keeping appointments, managing money, organizing and taking medications, planning, and recalling words or names [10]. Establishing a daily schedule and use of aids such as calendars and medication reminders greatly benefits those with MCI. This is the crucial stage to consider legal and financial decisions that will be faced in the future, as well. Assisting families and loved ones in finding support groups during this time can also provide a much needed connection to others dealing with similar situations.

While there are currently no curative pharmacological options for $\mathrm{AD}$, there are several prescriptions drugs available for the treatment of symptoms. Several medications in the class of cholinesterase inhibitors are used for mild to moderate $\mathrm{AD}$, which help to delay or prevent symptoms from becoming worse for a limited time [11]. These medications are Razadyne (galantamine), Exelon (rivastigmine), and Aricept (donepezil). It is believed that cholinesterase inhibitors prevent the breakdown of acetylcholine, a neurotransmitter involved in memory and cognition [11]. A patient's primary care physician or nurse practitioner can provide useful information regarding these medications, and educate regarding dosing, side effects, and length of effectiveness.

As the individual progressively declines, it is crucial for the healthcare team to update the plan of care to adapt to changing conditions and build upon elements that were introduced at the start of care. During this period, family and caregivers will face a variety of new tasks in the care of the patient, such as helping with activities of daily living, managing behavioral changes such as wandering or aggression, and finding resources for in-home help or assisted living care [3]. It was estimated that in 2014, family and other unpaid caregivers provided roughly 17.9 billion hours of unpaid care for individuals with Alzheimer's Disease [3]. Providing support and resources for family during this time is equally important as the medical management of the patient, and can be an area for great influence for nurses and social workers collaborating in the course of care. Safety concerns should also be incorporated into the plan of care during this time, as the risk for falls or wandering increases dramatically. Driving should be restricted, and outings should be made with a chaperone at all times.

With the progression of the disease, some changes in the pharmacological treatment may be indicated. For more severe $\mathrm{AD}$, the medication Namenda (memantine) is utilized. It is thought that memantine regulates glutamate, another neurotransmitter which can contribute to neuron death if found in excessive amounts [11]. The newly FDA approved medication Namzaric (donepezil and memantine) may also be used for moderate to severe AD. As behavioral symptoms worsen, the patient's pharmacological treatment may expand to include medications that can temporarily ease or limit symptoms such as anxiety, aggression, wandering, and insomnia. Antidepressants, anticonvulsants, sleep aids, anxiolytics, and antipsychotics can be integrated into the plan of care, but thorough teaching regarding medication management and administration at home must be provided to family and caregivers [11].

It is estimated that $61 \%$ of individuals with dementia have three or more comorbidities that require management [12]. Pneumonia, urinary tract infection, and congestive heart failure account for two-thirds of preventable hospital admissions for those with dementia [13]. Particular care must be taken by the primary health provider to offer education regarding the threat that existing or future comorbidities pose to the individual with AD. Providing family and caregivers information on interventions to prevent exacerbations of these conditions is a vital part of the evolving care plan for the management of Alzheimer's Disease and can increase patient comfort and quality of life.

The plan of care during the last stage of $\mathrm{AD}$ becomes focused on meeting basic care needs and supporting family in their caregiving. During this time, patients have lost most means of communication, have difficulty eating and swallowing, require total care involving mobility and basic 
personal functions, and are no longer able to remain continent [10]. Care options may need to be re-assessed during this time, as care for the patient becomes quite intensive. Referrals to assisting living facilities, in-home caregiver resources, and discussion about hospice care are appropriate interventions to include in the care plan. With the ability to swallow declining, pharmacotherapies may no longer be appropriate now, and medications for patient comfort available in sublingual or rectal forms may be recommended by the primary health provider. Continuing advocacy for safety and excellent daily care will assist in avoiding unnecessary hospitalization, and prevent complications regarding comorbidities, whether new or existing.

\section{Conclusion}

The toll that Alzheimer's is poised to take upon the American population poses a staggering challenge. With no curative treatments yet available, care management and symptom control remain the first line of defense. Family members look to healthcare professionals to provide them with guidance, support, and hope. Education and awareness of Alzheimer's Disease is a critical part of appropriate care delivery for health professionals, particularly those specializing in geriatrics. As research continues to search for better diagnostic methods and pharmacotherapies to delay or prevent the onset of $\mathrm{AD}$, the care plan for Alzheimer's patients must continue to focus on safety and quality of life. The genetic link to late-onset $\mathrm{AD}$ may very well be the answer for finding a cure, and as technology evolves, so will our knowledge. Perhaps one day, stories like those of Leslie Wynne will simply be memories of another disease conquered by innovation and discovery. That is, indeed, all of our hope.

\section{Appendix}

The Artwork of Leslie B. Wynne

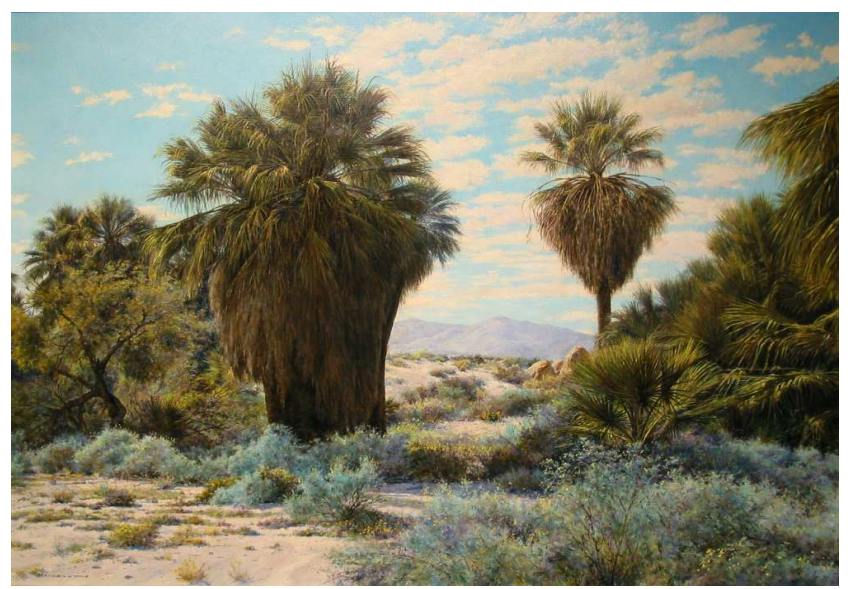

Figure 1. Oasis of Mara by Leslie Bernard Wynne Oil painting completed at height of career.

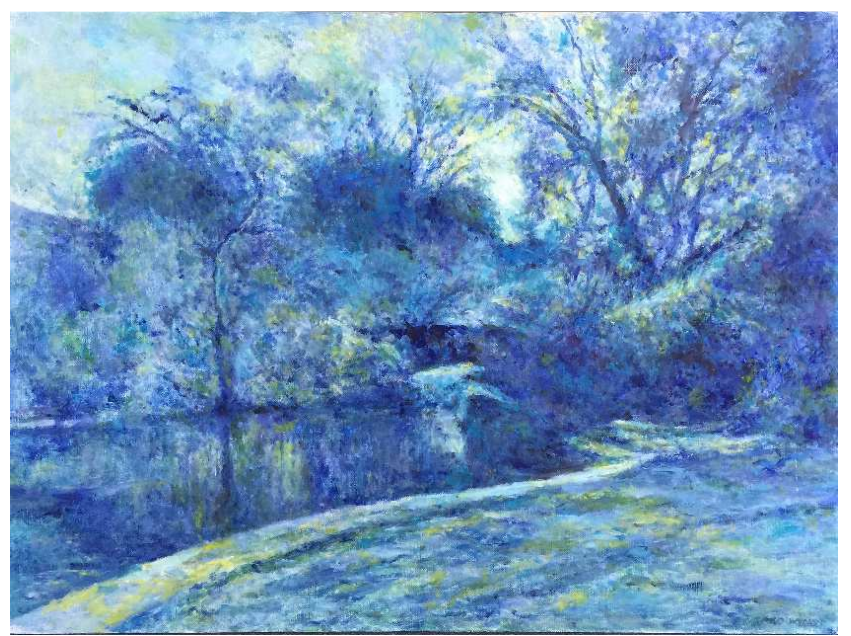

Figure 2. English Countryside by Leslie Bernard Wynne Oil painting completed during what family referred to as "the blue period".

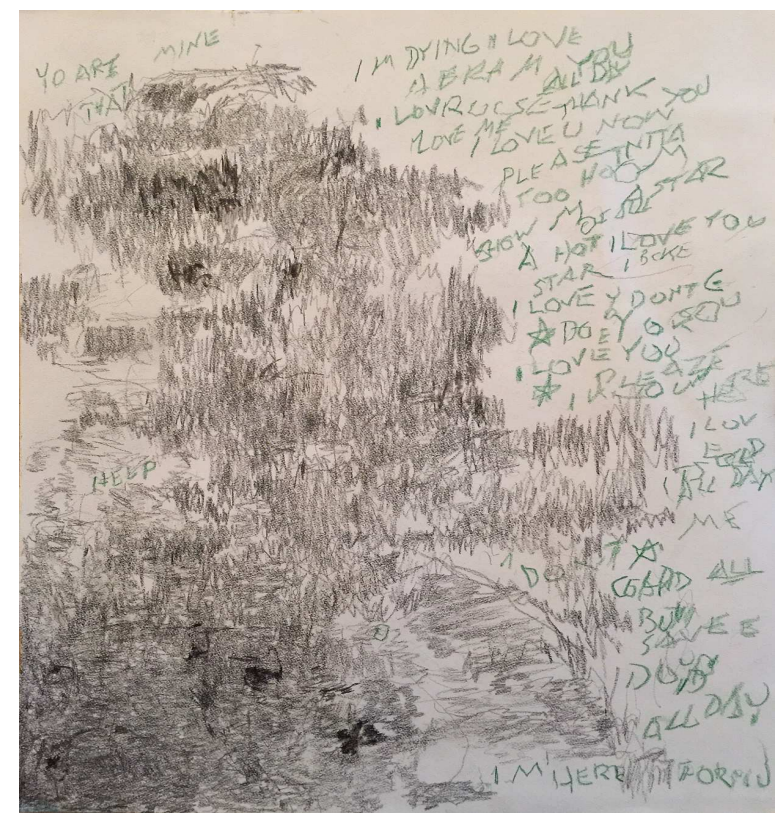

Figure 3. Sketch I by Leslie Bernard Wynne Pencil sketch completed during AD-related dementia.

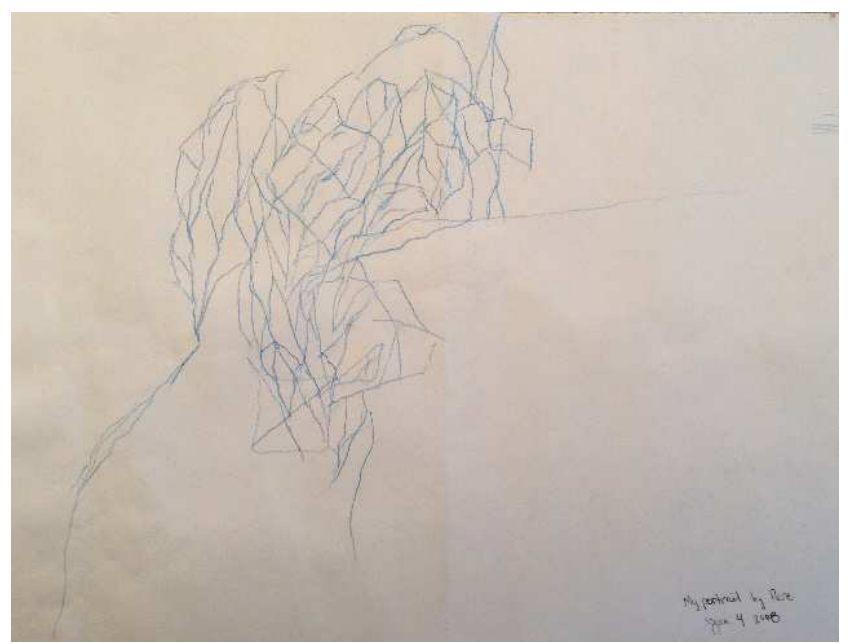

Figure 4. Sketch II by Leslie Bernard Wynne Completed January 4, 2008. 


\section{References}

[1] Hebert, L. E., Weuve, J., Scherr, P. A., \& Evans, D. A. (2013). Alzheimer disease in the United States (2010-2050) estimated using the 2010 census. Neurology, 80(19), 1778-1783. http://doi.org/10.1212/WNL.0b013e31828726f5

[2] Alois Alzheimer. (2015). Wikipedia. Retrieved from http://en.wikipedia.org/wiki/Alois_Alzheimer

[3] Alzheimer's Association. (2015a). 2015 Alzheimer's Disease facts and figures: Includes a special report on disclosing a diagnosis of Alzheimer's Disease. Retrieved from http://www.alz.org/facts/downloads/facts_figures_2015.pdf

[4] National Institute on Aging. (n.d.). About Alzheimer's Disease: Alzheimer's Basics. Retrieved from http://www.nia.nih.gov/alzheimers/topics/alzheimers-basics

[5] Alzheimer's Disease Education and Referral Center. (2011). Alzheimer's Disease genetics: Fact sheet. Bethesda, MD: National Institute of Health, Publication No. 11-6424.

[6] Holtzman, D. M., Herz, J., \& Bu, G. (2012). Apolipoprotein E and Apolipoprotein E Receptors: Normal Biology and Roles in Alzheimer Disease. Cold Spring Harbor Perspectives in Medicine, 2(3). http://doi.org/10.1101/cshperspect.a006312

[7] Loy, C. T., Schofield, P. R., Turner, A. M., \& Kwok, J. B. (2014). Genetics of dementia. The Lancet, 383(9919), 828-
840. http://doi.org/10.1016/S0140-6736(13)60630-3

[8] Goldman, J. S., Hahn, S. E., Catania, J. W., Larusse-Eckert, S., Butson, M. B., Rumbaugh, M., ... Bird, T. (2011). Genetic counseling and testing for Alzheimer disease: joint practice guidelines of the American College of Medical Genetics and the National Society of Genetic Counselors. Genetics in Medicine: Official Journal of the American College of Medical Genetics, 13(6), 597-605. http://doi.org/10.1097/GIM.0b013e31821d69b8

[9] Alzheimer's Disease Education and Referral Center. (2014). Assessing cognitive impairment in older patients: A quick guide for primary care physicians. Bethesda, MD: National Institute of Health.

[10] Alzheimer's Association. (2015b). Alzheimer's and Dementia Caregiver Center. Retrieved from http://www.alz.org/care/overview.asp

[11] Alzheimer's Disease Education and Referral Center. (2008). Alzheimer's Disease medications: Fact sheet. Bethesda, MD: National Institute of Health, Publication No. 08-3431.

[12] Fillit, H. M. (2000). The pharmacoeconomics of Alzheimer's disease. The American Journal of Managed Care, 6 (22 Suppl), S1139-1144; discussion S1145-1148.

[13] Phelan, E. A., Borson, S., Grothaus, L., Balch, S., \& Larson, E. B. (2012). Association of incident dementia with hospitalizations. JAMA, 307(2), 165-172. http://doi.org/10.1001/jama.2011.1964 